Groups Geom. Dyn. 8 (2014), 245-255

DOI $10.4171 / \mathrm{GGD} / 224$
Groups, Geometry, and Dynamics

(C) European Mathematical Society

\title{
Harmonic cochains and K-theory for $\tilde{A}_{2}$-groups
}

\author{
Guyan Robertson
}

\begin{abstract}
If $\Gamma$ is a torsion free $\tilde{A}_{2}$-group acting on an $\tilde{A}_{2}$ building $\Delta$, and $\mathfrak{U}_{\Gamma}$ is the associated boundary $C^{*}$-algebra, it is proved that $K_{0}\left(\mathfrak{U}_{\Gamma}\right) \otimes \mathbb{R} \cong \mathbb{R}^{2 \beta_{2}}$, where $\beta_{2}=\operatorname{dim}_{\mathbb{R}} H^{2}(\Gamma, \mathbb{R})$.
\end{abstract}

Mathematics Subject Classification (2010). 46L80; 58B34, 51E24, $20 \mathrm{G} 25$.

Keywords. Euclidean building, boundary, operator algebra.

\section{Introduction}

Let $\Gamma$ be an $\tilde{A}_{2}$-group acting on an $\tilde{A}_{2}$ building $\Delta$ of order $q$. The Furstenberg boundary $\Omega$ of $\Delta$ is the set of chambers of the spherical building at infinity, endowed with a natural compact totally disconnected topology. The topological action of $\Gamma$ on $\Omega$ is encoded in the full crossed product $C^{*}$-algebra $\mathfrak{A}_{\Gamma}=C(\Omega) \rtimes \Gamma$, which is studied in [10], [11], [12]. This full crossed product is isomorphic to the reduced crossed product, since the action of $\Gamma$ on $\Omega$ is amenable [10], Section 4.2. As the notation suggests, $\mathfrak{U}_{\Gamma}$ depends only on $\Gamma$ [12]. Motivated by rigidity theorems of Mostow, Margulis and others, whose proofs rely on the study of boundary actions, it is of interest to determine the extent to which the boundary $C^{*}$-algebra $\mathfrak{U}_{\Gamma}$ determines the group $\Gamma$.

In [12], T. Steger and the author computed the K-theory of $\mathfrak{U}_{\Gamma}$ for many $\tilde{A}_{2}$-groups with $q \leq 13$. The computations were done for all the $\tilde{A}_{2}$-groups in the cases $q=2,3$ and for several representative groups for each of the other values of $q \leq 13$. If $q=2$ there are precisely eight $\tilde{A}_{2}$-groups $\Gamma$, all of which embed as lattices in PGL $(3, \mathbb{K})$, where $\mathbb{K}=\mathbb{F}_{2}((X))$ or $\mathbb{K}=\mathbb{Q}_{2}$. If $q=3$ there are 89 possible $\tilde{A}_{2}$-groups, of which 65 are "exotic" in the sense that they do not embed naturally in linear groups. Exotic $\tilde{A}_{n}$-groups only exist if $n=2$, since all locally finite Euclidean buildings of dimension $\geq 3$ are associated to linear algebraic groups. This justifies, to some extent, the focus on $\tilde{A}_{2}$-groups.

For each $\tilde{A}_{2}$-group $\Gamma$, the $C^{*}$-algebra $\mathfrak{U}_{\Gamma}$ has the structure of a rank-2 CuntzKrieger algebra [11], Theorem 7.7. These algebras are classified up to isomorphism 
by their $K$-groups [11], Remark 6.5, and it was proved in Theorem 2.1 of [12] that

$$
K_{0}\left(\mathfrak{A}_{\Gamma}\right)=K_{1}\left(\mathfrak{A}_{\Gamma}\right)=\mathbb{Z}^{2 r} \oplus T,
$$

where $r \geq 0$ and $T$ is a finite group. The computations in [12] led to some striking observations. For example, the three torsion-free $\tilde{A}_{2}$ subgroups of $\operatorname{PGL}_{3}\left(\mathbb{Q}_{2}\right)$ are distinguished from each other by $K_{0}\left(\mathfrak{U}_{\Gamma}\right)$. There was also evidence for the conjecture that, for any torsion free $\tilde{A}_{2}$-group $\Gamma$, the integer $r$ in the equation (1) is equal to the second Betti number of $\Gamma$. The purpose of this article is to prove that this is indeed the case.

Theorem 1.1. If $\Gamma$ is a torsion free $\tilde{A}_{2}$-group acting on an $\tilde{A}_{2}$ building $\Delta$ of order q, then

$$
K_{0}\left(\mathfrak{A}_{\Gamma}\right) \otimes \mathbb{R} \cong \mathbb{R}^{2 \beta_{2}},
$$

where $\beta_{2}=\operatorname{dim}_{\mathbb{R}} H^{2}(\Gamma, \mathbb{R})=\frac{1}{3}(q-2)\left(q^{2}+q+1\right)$.

The article [12] identified the integer $r$ in (1) with the rank of a certain finitely generated abelian group $C(\Gamma)$. Two new ideas lead to the proof of Theorem 1.1. The local structure of the building $\Delta$, together with the fact that $\Gamma$ has Kazhdan's property (T), is used to show that $C(\Gamma) \otimes \mathbb{R}$ is isomorphic to the space of $\Gamma$-invariant $\mathbb{R}$-valued cochains on $\Delta$, in the sense of [1], [4]. Then, according to an isomorphism of Garland [5], this space is isomorphic to $H^{2}(\Gamma, \mathbb{R})$.

Remark 1.2. An $\tilde{A}_{2}$-group is a natural analogue of a free group, which acts freely and transitively on the vertex set of a tree (which is a building of type $\tilde{A}_{1}$ ). If the tree is homogeneous of degree $q+1$, with $q \geq 2$, then $\Gamma$ is a free group on $\frac{1}{2}(q+1)$ generators and one can again form the full crossed product $C^{*}$-algebra $\mathfrak{A}_{\Gamma}=C(\Omega) \rtimes \Gamma$, where $\Omega$ is the space of ends of the tree. The analogue of Theorem 1.1 states that $K_{0}\left(\mathfrak{U}_{\Gamma}\right) \otimes \mathbb{R} \cong \mathbb{R}^{\beta_{1}}$, where $\beta_{1}=\operatorname{dim}_{\mathbb{R}} H^{1}(\Gamma, \mathbb{R})=\frac{1}{2}(q+1)$ [8], Theorem 1 .

Remark 1.3. Another simple $C^{*}$-algebra associated with the $\tilde{A}_{2}$-group $\Gamma$ is the reduced group $C^{*}$-algebra $C_{r}^{*}(\Gamma)$. It is shown in [9], Theorem 6.1, that $K_{0}\left(C_{r}^{*}(\Gamma)\right)=$ $\mathbb{Z}^{\chi(\Gamma)}$. This is a consequence of the fact that $\tilde{A}_{2}$-groups belong to the class of groups for which the Baum-Connes conjecture is known to be true.

Remark 1.4. This paper is a sequel to the articles [11], [12]. The key results used are [11], Theorem 7.7, which shows that $\mathfrak{A}_{\Gamma}$ is isomorphic to a rank-2 Cuntz-Krieger algebra, and [12], Theorem 2.1, which shows that the $K$-theory of this algebra is given by equation (1).

What happens in the case of a torsion free $\tilde{A}_{n}$-group $\Gamma(n \geq 3)$ ? There seems to be no fundamental obstruction to generalising [11], Theorem 7.7, to identify the boundary crossed product algebra with a higher rank Cuntz-Krieger algebra, in the sense of [11]. The arguments of the present paper should also generalise, but additional conditions which are vacuous in the rank-2 case would need to be verified [1], 
Theorem 2.3 (C), (D). However it would be more difficult to generalise [12], Theorem 2.1. This is because the proof of that result uses a Kasparov spectral sequence [12], Proposition 4.1, whose limit is clear only in the rank-2 case.

\section{2. $\tilde{A}_{2}$-groups}

Consider a locally finite building $\Delta$ of type $\tilde{A}_{2}$. Each vertex $v$ of $\Delta$ has a type $\tau(v) \in \mathbb{Z} / 3 \mathbb{Z}$, and each chamber (maximal simplex) of $\Delta$ has exactly one vertex of each type. Each edge $e$ is directed, with initial vertex of type $i$ and final vertex of type $i+1$. An automorphism $\alpha$ of $\Delta$ is type rotating if there exists $i \in \mathbb{Z} / 3 \mathbb{Z}$ such that $\tau(\alpha(v))=\tau(v)+i$ for all vertices $v$ of $\Delta$.

Suppose that $\Gamma$ is a group of type rotating automorphisms of $\Delta$, which acts freely and transitively on the vertex set of $\Delta$. Such a group is called an $\tilde{A}_{2}$-group. The theory of $\tilde{A}_{2}$-groups has been developed in [3] and some, but not all, $\tilde{A}_{2}$-groups embed as lattice subgroups of $\mathrm{PGL}_{3}(\mathbb{K})$. Any $\tilde{A}_{2}$-group can be constructed as follows [3], I, Section 3. Let $(P, L)$ be a finite projective plane of order $q$. There are $q^{2}+q+1$ points (elements of $P$ ) and $q^{2}+q+1$ lines (elements of $L$ ). Let $\lambda: P \rightarrow L$ be a bijection and write $\lambda(\xi)=\bar{\xi}$. A triangle presentation compatible with $\lambda$ is a set $\mathcal{T}$ of ordered triples $\left(\xi_{i}, \xi_{j}, \xi_{k}\right)$ where $\xi_{i}, \xi_{j}, \xi_{k} \in P$, with the following properties.

(i) Given $\xi_{i}, \xi_{j} \in P$, then $\left(\xi_{i}, \xi_{j}, \xi_{k}\right) \in \mathcal{T}$ for some $\xi_{k} \in P$ if and only if $\xi_{j}$ and $\bar{\xi}_{i}$ are incident, i.e. $\xi_{j} \in \bar{\xi}_{i}$.

(ii) $\left(\xi_{i}, \xi_{j}, \xi_{k}\right) \in \mathcal{T} \Rightarrow\left(\xi_{j}, \xi_{k}, \xi_{i}\right) \in \mathcal{T}$.

(iii) Given $\xi_{i}, \xi_{j} \in P$, then $\left(\xi_{i}, \xi_{j}, \xi_{k}\right) \in \mathcal{T}$ for at most one $\xi_{k} \in P$.

In [3] there is exhibited a complete list of triangle presentations for $q=2$ and $q=3$. Given a triangle presentation $\mathcal{T}$, one can form the group

$$
\left.\Gamma=\Gamma_{\mathcal{T}}=\langle P| \xi_{i} \xi_{j} \xi_{k}=1 \text { for }\left(\xi_{i}, \xi_{j}, \xi_{k}\right) \in \mathcal{T}\right\rangle
$$

The Cayley graph of $\Gamma$ with respect to the generating set $P$ is the 1 -skeleton of a building $\Delta$ of type $\tilde{A}_{2}$. Vertices are elements of $\Gamma$ and a directed edge of the form $(\gamma, \gamma \xi)$ with $\gamma \in \Gamma$ is labeled by the generator $\xi \in P$.

The link of a vertex $\gamma$ of $\Delta$ is the incidence graph of the projective plane $(P, L)$, where the lines in $L$ correspond to the inverses in $\Gamma$ of the generators in $P$. In other words, $\bar{\xi}=\xi^{-1}$ for $\xi \in P$.

For the rest of this article, $\Gamma$ is assumed to be torsion free. Therefore $\Gamma$ acts freely on $\Delta$ and $X=\Gamma \backslash \Delta$ is a 2-dimensional cell complex with universal covering $\Delta$. Let $X^{k}$ denote the set of oriented $k$-cells of $X$ for $k=0,1,2$. Thus $X^{1}$ may be identified with $P$ and $X^{2}$ may be identified with the set of orbits of elements of $\mathcal{T}$ under cyclic permutations.

Let $\widehat{\Delta}^{2}$ be the directed version of $\Delta^{2}$ in which each 2-simplex has a specified base vertex, so that $\mathbb{Z} / 3 \mathbb{Z}$ acts naturally on $\widehat{\Delta}^{2}$. Let $\widehat{X}^{2}:=\widehat{\Delta}^{2} / \Gamma$, the set of directed 


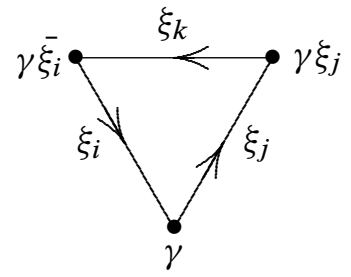

Figure 1. A chamber based at a vertex $\gamma$.

2-cells of $X$. Then $\hat{X}^{2}$ may be identified with $\mathcal{T}$. From now on $a=\left\langle a_{0}, a_{1}, a_{2}\right\rangle$ will denote an element of $\mathcal{T}$, regarded as a directed 2-cell. Figure 2 illustrates the three directed 2-cells associated with an oriented 2-cell of $X$. In the diagram, the 2 -cells are thought of as being directed upwards and the symbol $\bullet$ is placed opposite the "top" edge to indicate that direction.
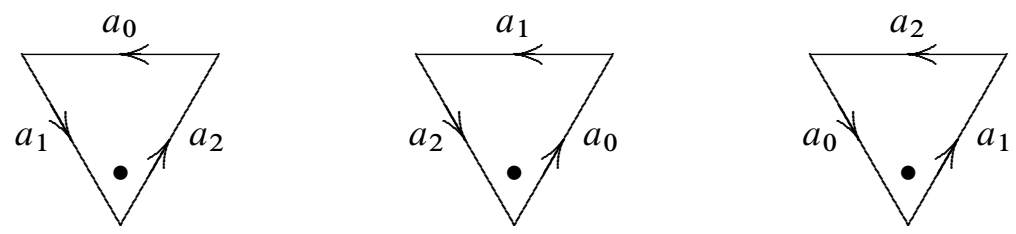

Figure 2. The directed 2-cells $\left\langle a_{0}, a_{1}, a_{2}\right\rangle,\left\langle a_{1}, a_{2}, a_{0}\right\rangle,\left\langle a_{2}, a_{0}, a_{1}\right\rangle$.

\section{K-theory}

Transition matrices $M=\left(m_{a b}\right)_{a, b \in \hat{X}^{2}}$ and $N=\left(n_{a b}\right)_{a, b \in \hat{X}^{2}}$ are defined as follows. If $a, b \in \hat{X}^{2}$ then $m_{a b}=1$ if and only if there are labeled triangles representing $a, b$ in the building $\Delta$ which lie as shown on the right of Figure 3. If no such diagram is possible then $m_{a b}=0$.

In terms of the projective plane $(P, L)$, the matrix $M$ is defined by

$$
m_{a b}=1 \Longleftrightarrow b_{2} \notin \bar{a}_{2}, \bar{b}_{1}=a_{0} \vee b_{2} .
$$

It follows that each row or column of $M$ has precisely $q^{2}$ nonzero entries.

Similarly, the matrix $N$ is defined by

$$
n_{a c}=1 \Longleftrightarrow a_{1} \notin \bar{c}_{1}, c_{2}=\bar{a}_{0} \wedge \bar{c}_{1} .
$$

as illustrated on the left of Figure 3.

Let $r$ be the rank, and $T$ the torsion part, of the abelian group $C(\Gamma)$ with generating set $\widehat{X}^{2}$ and relations 

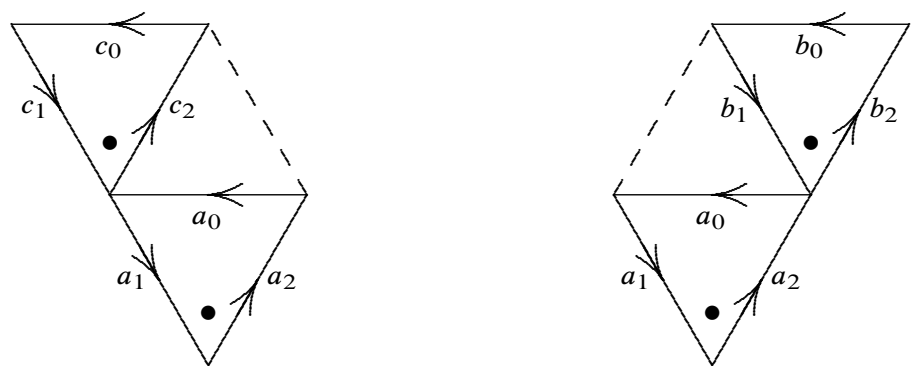

Figure 3. The relations $n_{a c}=1$ and $m_{a b}=1$.

$$
a=\sum_{b \in \hat{X}^{2}} m_{a b} b=\sum_{b \in \hat{X}^{2}} n_{a b} b, \quad a \in \widehat{X}^{2} .
$$

Thus $C(\Gamma) \cong \mathbb{Z}^{r} \oplus T$. The following result was proved in [12], Theorem 2.1.

Theorem 3.1. Let $\Gamma$ be an $\tilde{A}_{2}$-group, and let $r$ be the rank, and $T$ the torsion part of $C(\Gamma)$. Then

$$
K_{0}\left(\mathfrak{U}_{\Gamma}\right)=K_{1}\left(\mathfrak{U}_{\Gamma}\right)=\mathbb{Z}^{2 r} \oplus T
$$

Given $\xi \in P$, let

$$
\langle\xi\rangle=\sum_{\substack{a \in \hat{X}^{2} \\ a_{2}=\xi}} a \in C(\Gamma) .
$$

It is sometimes convenient to write such sums pictorially as

$$
\langle\xi\rangle=\sum_{\sqrt{a} / \xi} a .
$$

Note that $a \in \hat{X}^{2}$ with $a_{2}=\xi$ if and only if $a=\left\langle a_{0}, a_{1}, a_{2}\right\rangle$ where $a_{2}=\xi$ and $a_{0} \in \bar{\xi}$ (and $a_{1}$ is then uniquely determined). There are $q+1$ such choices of $a_{0}$ and so there are $q+1$ terms in the sum (6). Similar remarks apply to the element $\langle\bar{\xi}\rangle \in C(\Gamma)$ defined by

$$
\langle\bar{\xi}\rangle=\sum_{\substack{a \in \hat{X}^{2} \\ a_{1}=\xi}} a=\sum_{\xi} a .
$$

In what follows the element

$$
\varepsilon=\sum_{a \in \widehat{X}^{2}} a
$$

plays a special role. An important observation, which is needed subsequently, is that $\varepsilon$ has finite order in $C(\Gamma)$. The statement and its proof are very like [12], Proposition 8.2. 
Lemma 3.2. In the group $C(\Gamma),\left(q^{2}-1\right) \varepsilon=0$.

Proof. Using relations (4) and the fact that each column of the matrix $M$ has precisely $q^{2}$ nonzero entries,

$$
\varepsilon=\sum_{a \in \widehat{X}^{2}} a=\sum_{a \in \hat{X}^{2}} \sum_{b \in \hat{X}^{2}} m_{a b} b=\sum_{b \in \hat{X}^{2}}\left(\sum_{a \in \widehat{X}^{2}} m_{a b}\right) b=\sum_{b \in \hat{X}^{2}} q^{2} b=q^{2} \varepsilon .
$$

Lemma 3.3. If $\left\langle a_{0}, a_{1}, a_{2}\right\rangle \in \hat{X}^{2}$ then, in the group $C(\Gamma)$,

$$
\begin{aligned}
\left\langle a_{1}\right\rangle-\left\langle a_{2}, a_{0}, a_{1}\right\rangle & =\left\langle\bar{a}_{2}\right\rangle-\left\langle a_{1}, a_{2}, a_{0}\right\rangle ; \\
\left\langle a_{0}\right\rangle+\left\langle a_{1}\right\rangle+\left\langle a_{2}\right\rangle & =\varepsilon .
\end{aligned}
$$

Proof. Fix the base vertex $1 \in \Delta$. Any generator $a$ for $C(\Gamma)$ has a unique representative directed chamber $\sigma_{a}$ based at 1 . The chamber $\sigma_{a}$ has vertices $1, a_{1}^{-1}, a_{2}$. By [6], Section 15.4, each chamber based at 1, other than $\sigma_{a}$ lies in a common apartment with $\sigma_{a}$, in exactly one of the five positions $\tau_{2}, \tau_{3}, \tau_{4}, \tau_{5}, \tau_{6}$ in Figure 4 . As before, directed chambers will be pointed.

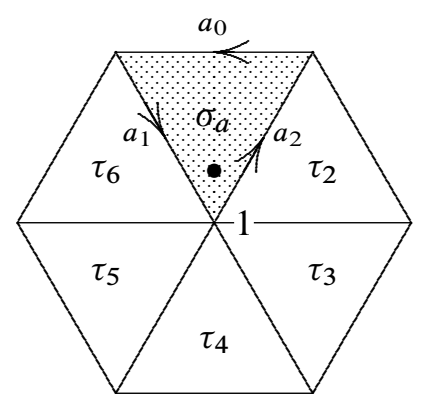

Figure 4

The left side of (9a) is equal to the sum of all the elements $b \in \widehat{X}^{2}$ represented by directed chambers $\sigma_{b}$ in position $\tau_{6}$, as illustrated in Figure 5 (a). Each such element $b$ satisfies $b_{2}=a_{1}$, and the relations (4) imply that

$$
b=\sum_{c \in \widehat{X}^{2}} m_{b c} c .
$$

That is, $b$ is the sum of all the elements $c \in \widehat{X}^{2}$ with representative directed chambers $\sigma_{c}$ lying in position $\tau_{4}$, as illustrated in Figure 5 (a). Moreover, if $\sigma_{c}$ is any directed chamber with base vertex 1 , lying in position $\tau_{4}$, then it arises in this way from a unique chamber $\sigma_{b}$ in position $\tau_{6}$. To see this, it is enough to take the convex hull 
of any such chamber $\sigma_{c}$ with $\sigma_{a}$, which completely determines the whole hexagon in Figure 5 (a). Therefore the left side of (9a) is equal to the sum of all the elements $c \in \widehat{X}^{2}$ represented by directed chambers $\sigma_{c}$ based at 1 which lie in position $\tau_{4}$ of Figure 4 .

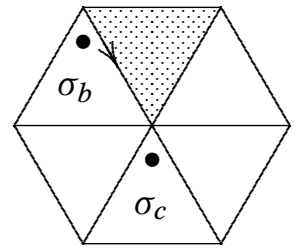

(a)

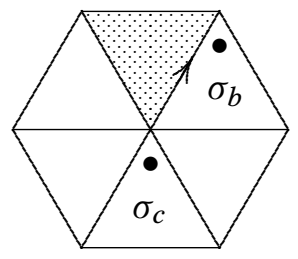

(b)

Figure 5

Similarly, the right side of (9a) is equal to the sum of all the elements $b \in \hat{X}^{2}$ represented by directed chambers $\sigma_{b}$ in position $\tau_{2}$ as illustrated in Figure 5 (b). The relations (4) imply that, for each such chamber $b$,

$$
b=\sum_{b \in \widehat{X}^{2}} n_{a c} c .
$$

It follows that the right side of (9a) is also equal to the sum of all the elements $c \in \hat{X}^{2}$ represented by directed chambers based at 1 which lie in position $\tau_{4}$ of Figure 4 . This proves that the left and right sides of (9a) are equal.

The next task is to prove (9b). Recall that $\varepsilon$ is the sum of all the elements of $\hat{X}^{2}$, and representative directed chambers for elements of this sum are $\sigma_{a}$ together with all chambers lying in any of the five positions $\tau_{2}, \tau_{3}, \tau_{4}, \tau_{5}, \tau_{6}$ in Figure 4 .

The set of chambers based at the vertex 1 representing the elements of the sum $\left\langle a_{2}\right\rangle$ consists of $\sigma_{a}$ together with all directed chambers lying in the position $\tau_{2}$, as illustrated in Figure 6 (a). Here it may also be convenient to refer back to equation (7).

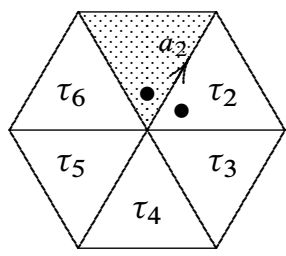

(a)

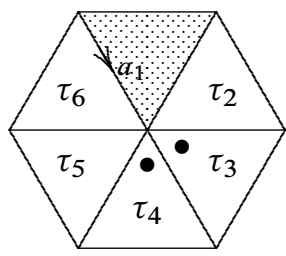

(b)

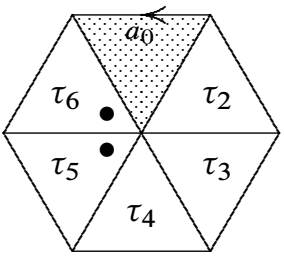

(c)

Figure 6 
Using the relations (4), the sum $\left\langle a_{1}\right\rangle$ is equal to the sum of elements represented by chambers lying in the position $\tau_{3}$ or $\tau_{4}$, as in Figure $6(\mathrm{~b})$.

Finally, the sum $\left\langle a_{0}\right\rangle$ is equal to the sum of elements represented by directed chambers lying in the position $\tau_{5}$ or $\tau_{6}$, as in Figure 6 (c). For, cyclically permuting the indices in the equation ( 9 a) gives

$$
\left\langle a_{0}\right\rangle-\left\langle a_{1}, a_{2}, a_{0}\right\rangle=\left\langle\bar{a}_{1}\right\rangle-\left\langle a_{0}, a_{1}, a_{2}\right\rangle
$$

Therefore $\left\langle a_{0}\right\rangle=\left\langle a_{1}, a_{2}, a_{0}\right\rangle+\left\langle\bar{a}_{1}\right\rangle-\left\langle a_{0}, a_{1}, a_{2}\right\rangle$. Referring to Figure 6 (c), the relations (4) show that $\left\langle a_{1}, a_{2}, a_{0}\right\rangle$ is the sum of elements represented by directed chambers in position $\tau_{5}$. Also $\left\langle\bar{a}_{1}\right\rangle-\left\langle a_{0}, a_{1}, a_{2}\right\rangle$ is the sum of elements represented by directed chambers in position $\tau_{6}$.

This completes the proof that $\left\langle a_{0}\right\rangle+\left\langle a_{1}\right\rangle+\left\langle a_{2}\right\rangle=\varepsilon$.

The next lemma is a major step in the proof of the main theorem. It depends on the fact that $\Gamma$ has Kazhdan's property (T), which in turn depends only on the local structure of the building $\Delta$. See, for example, the proof of [2], Theorem 5.7.7.

Lemma 3.4. In the group $C(\Gamma) \otimes \mathbb{R}$, for all $\left\langle a_{0}, a_{1}, a_{2}\right\rangle \in \hat{X}^{2}$ and $\xi \in P$,

$$
\begin{aligned}
& \left\langle a_{0}, a_{1}, a_{2}\right\rangle \otimes 1=\left\langle a_{1}, a_{2}, a_{0}\right\rangle \otimes 1=\left\langle a_{2}, a_{0}, a_{1}\right\rangle \otimes 1 ; \\
& \langle\xi\rangle \otimes 1=0=\langle\bar{\xi}\rangle \otimes 1 .
\end{aligned}
$$

Proof. By Lemma 3.2, $\varepsilon$ has finite order in $C(\Gamma)$ and hence $\varepsilon \otimes 1$ is zero in $C(\Gamma) \otimes \mathbb{R}$. Therefore, by (9b),

$$
\left\langle a_{0}\right\rangle \otimes 1+\left\langle a_{1}\right\rangle \otimes 1+\left\langle a_{2}\right\rangle \otimes 1=0
$$

for $\left\langle a_{0}, a_{1}, a_{2}\right\rangle \in \hat{X}^{2}$. It follows from the presentation of $\Gamma$ that the map $\xi \mapsto\langle\xi\rangle \otimes 1$, $\xi \in P$, induces a homomorphism $\theta$ from $\Gamma$ into the abelian group $C(\Gamma) \otimes \mathbb{R}$.

The $\tilde{A}_{2}$-group $\Gamma$ has Kazhdan's property (T), by [2], Theorem 5.7.7. It follows that the range of $\theta$ is finite [2], Corollary 1.3.6, and hence zero, since $C(\Gamma) \otimes \mathbb{R}$ is torsion free. Therefore $\langle\xi\rangle \otimes 1=0, \xi \in P$. Similarly, $\langle\bar{\xi}\rangle \otimes 1=0, \xi \in P$. This proves (10b). The relation (9a) then implies that $\left\langle a_{1}, a_{2}, a_{0}\right\rangle \otimes 1=\left\langle a_{2}, a_{0}, a_{1}\right\rangle \otimes 1$ and the rest of (10a) follows by symmetry.

Let $C_{0}(\Gamma)$ be the abelian group with generating set $\widehat{X}^{2}$ and the following relations:

$$
\begin{aligned}
& \left\langle a_{0}, a_{1}, a_{2}\right\rangle=\left\langle a_{1}, a_{2}, a_{0}\right\rangle=\left\langle a_{2}, a_{0}, a_{1}\right\rangle, \quad\left\langle a_{0}, a_{1}, a_{2}\right\rangle \in \hat{X}^{2} \\
& \langle\xi\rangle=0=\langle\bar{\xi}\rangle, \quad \xi \in P .
\end{aligned}
$$

Lemma 3.5. The relations (11) imply the relations (4). 
Proof. Let $a=\left\langle a_{0}, a_{1}, a_{2}\right\rangle \in \hat{X}^{2}$. Then, using the relations (11), and referring to Figure 7,

$$
\begin{aligned}
a & =\left\langle a_{0}, a_{1}, a_{2}\right\rangle=\left\langle a_{1}, a_{2}, a_{0}\right\rangle \quad[\operatorname{using}(11 \mathrm{a})] \\
& =-\sum_{\substack{\left\langle c_{2}, b_{1}, a_{0}\right\rangle \in \hat{X}^{2} \\
c_{2} \neq a_{1}}}\left\langle c_{2}, b_{1}, a_{0}\right\rangle \quad\left[\text { using (11b), with } \xi=a_{0}\right] \\
& =-\sum_{\substack{\left\langle c_{2}, b_{1}, a_{0}\right\rangle \in \hat{X}^{2} \\
c_{2} \neq a_{1}}}\left(-\sum_{\substack{\left\langle b_{0}, b_{1}, b_{2}\right\rangle \in \hat{X}^{2} \\
b_{0} \neq c_{2}}}\left\langle b_{0}, b_{1}, b_{2}\right\rangle\right) \quad \text { [using (11b) again] } \\
& =\sum_{b \in \hat{X}^{2}} m_{a b} b .
\end{aligned}
$$

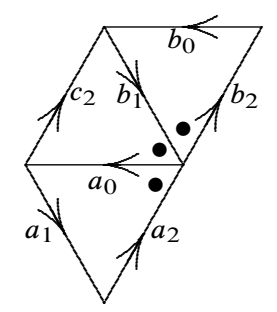

Figure 7

The proof of the relations $a=\sum_{b \in \widehat{X}^{2}} n_{a b} b$ in (4) is similar.

Proposition 3.6. If $\Gamma$ is a torsion free $\tilde{A}_{2}$-group, then $C(\Gamma) \otimes \mathbb{R}=C_{0}(\Gamma) \otimes \mathbb{R}$.

Proof. The groups have the same set of generators. By Lemmas 3.4 and 3.5, the relations in each group imply the relations in the other. The groups are therefore equal.

\section{Harmonic cochains and proof of the main result}

A harmonic 2-cochain [4] is a function $c: \widehat{X}^{2} \rightarrow \mathbb{R}$ satisfying the following conditions for all $a \in \widehat{X}^{2}$ and for all $\xi \in P$.

$$
\begin{aligned}
c\left(\left\langle a_{0}, a_{1}, a_{2}\right\rangle\right) & =c\left(\left\langle a_{1}, a_{2}, a_{0}\right\rangle\right)=c\left(\left\langle a_{2}, a_{0}, a_{1}\right\rangle\right) ; \\
c(\langle\xi\rangle) & =c(\langle\bar{\xi}\rangle)=0 .
\end{aligned}
$$

Denote the set of harmonic 2-cochains by $C_{\text {har }}^{2}\left(\widehat{X}^{2}\right)$. Since the group $\Gamma$ acts freely on $\Delta, C_{\text {har }}^{2}\left(\hat{X}^{2}\right)$ may be identified with the space of $\Gamma$-invariant harmonic cochains $c: \widehat{\Delta}^{2} \rightarrow \mathbb{R}$, in the sense of [1]. Now $C_{\text {har }}^{2}\left(\hat{X}^{2}\right)$ is the algebraic dual of $C_{0}(\Gamma) \otimes \mathbb{R}$. The next result is therefore an immediate consequence of Proposition 3.6. 
Proposition 4.1. $C_{\mathrm{har}}^{2}\left(\widehat{X}^{2}\right)$ is isomorphic to $C(\Gamma) \otimes \mathbb{R}$.

The proof of Theorem 1.1 can now be completed. By Theorem 3.1, it is sufficient to show that the rank $r$ of $C(\Gamma)$ is equal to $\beta_{2}=\operatorname{dim}_{\mathbb{R}} H^{2}(\Gamma, \mathbb{R})$. Garland's isomorphism [1], Section 3.1, states that $H^{2}(\Gamma, \mathbb{R}) \cong C_{\text {har }}^{2}\left(\hat{X}^{2}\right)$. Note that the account of Garland's Theorem in [1] relates to the case where $\Gamma$ is a lattice in $\operatorname{PGL}(3, \mathbb{K})$, but the proof applies without change to all torsion free $\tilde{A}_{2}$-groups.

It follows from Proposition 4.1 that $C(\Gamma) \otimes \mathbb{R} \cong H^{2}(\Gamma, \mathbb{R})$. Theorem 3.1 now implies that $K_{0}\left(\mathfrak{U}_{\Gamma}\right) \otimes \mathbb{R} \cong \mathbb{R}^{2 \beta_{2}}$.

It remains to identify $\beta_{2}$ explicitly. The Euler characteristic of $\Gamma$ is $\chi(\Gamma)=$ $\frac{1}{3}(q-1)\left(q^{2}-1\right)[7]$, Section 4. Now $\chi(\Gamma)=\beta_{0}-\beta_{1}+\beta_{2}$ where $\beta_{i}=$ $\operatorname{dim}_{\mathbb{R}} H_{i}(\Gamma, \mathbb{R})$. Since $\Gamma$ has Kazhdan's property $(\mathrm{T})$, the abelianisation $\Gamma /[\Gamma, \Gamma]$ is finite [2], Corollary 1.3.6, and so $\beta_{1}=0$. Also $\beta_{0}=1$. Therefore $\beta_{2}=$ $\chi(\Gamma)-1=\frac{1}{3}(q-2)\left(q^{2}+q+1\right)$. This completes the proof.

\section{References}

[1] G. Alon and E. de Shalit, Cohomology of discrete groups in harmonic cochains on buildings. Israel J. Math. 135 (2003), 355-380. Zbl 1073.14027 MR 1997050

[2] B. Bekka, P. de la Harpe, and A. Valette, Kazhdan's property (T), New Math. Monogr. 11, Cambridge University Press, Cambridge, 2008. Zbl 1146.22009 MR 2415834

[3] D. I. Cartwright, A. M. Mantero, T. Steger, and A. Zappa, Groups acting simply transitively on the vertices of a building of type $\tilde{A}_{2}$. I. Geom. Dedicata 47 (1993), 143-166. Zbl 0784.51010 MR 1232965

[4] B. Eckmann, Introduction to $\ell_{2}$-methods in topology: reduced $\ell_{2}$-homology, harmonic chains, $\ell_{2}$-Betti numbers. Israel J. Math. 117 (2000), 183-219. Zbl 0948.55006 MR 1760592

[5] H. Garland, $p$-adic curvature and the cohomology of discrete subgroups of $p$-adic groups. Ann. of Math. (2) 97 (1973), 375-423. Zbl 0262.22010 MR 0320180

[6] P. Garrett, Buildings and classical groups. Chapman \& Hall, London 1997. Zbl 0933.20019 MR 1449872

[7] G. Robertson, Torsion in $K$-theory for boundary actions on affine buildings of type $\tilde{A}_{n}$. K-Theory 22 (2001), 251-269. Zbl 0980.46052 MR 1837234

[8] G. Robertson, Boundary operator algebras for free uniform tree lattices. Houston J. Math. 31 (2005), 913-935. Zbl 1093.46040 MR 2148805

[9] G. Robertson, Torsion in boundary coinvariants and $K$-theory for affine buildings. $K$ Theory 33 (2005), 347-369. Zbl 1079.51007 MR 2220525

[10] G. Robertson and T. Steger, $C^{*}$-algebras arising from group actions on the boundary of a triangle building. Proc. London Math. Soc. (3) 72 (1996), 613-637. Zbl 0869.46035 MR 1376771

[11] G. Robertson and T. Steger, Affine buildings, tiling systems and higher rank Cuntz-Krieger algebras. J. Reine Angew. Math. 513 (1999), 115-144. Zbl 1064.46504 MR 1713322 
[12] G. Robertson and T. Steger, Asymptotic $K$-theory for groups acting on $\tilde{\mathrm{A}}_{2}$ buildings. Canad. J. Math. 53 (2001), 809-833. Zbl 0993.46039 MR 1848508

Received October 4, 2011; revised September 13, 2012

G. Robertson, School of Mathematics and Statistics, University of Newcastle, Newcastle upon Tyne, NE1 7RU, U.K.

E-mail: guyanrobertson@gmx.com 